\title{
Marital Transitions and Depressive Symptoms among Older Adults: Examining Educational Differences
}

\author{
Claudia Recksiedler ${ }^{a, b}$ Robert S. Stawski ${ }^{c}$ \\ a Department for Social Monitoring and Methodology, German Youth Institute, Munich, Germany; \\ ${ }^{b}$ NCCR LIVES, Chavannes-près-Renens, Switzerland; ' School of Social and Behavioral Health Sciences, \\ Oregon State University, Corvallis, OR, USA
}

\section{Keywords}

Life events · Depression · Education · Social gradient .

Ageing societies $\cdot$ Health and Retirement Study

\begin{abstract}
Background: Later decades of the life course have undergone rapid transformations due to demographic changes in ageing societies, such as more frequent occurrences of laterlife marital transitions. Adaption to these transitions, even when welcomed, brings novel chances and challenges in negotiating new social roles in old age, which could reinforce preexisting disparities in the acquisition and mastery of resources, social ties, and coping strategies. Objectives: Because the ability to weather later-life marital transitions may depend on the long arm of education acquired earlier in the life course, the present study aims to identify and track trends in the prevalence of marriage, divorce/separation, and widowhood among sociodemographic subgroups; link the occurrence of those transitions with mental health; and test the influence of educational attainment on these associations. Methods: We employ an intraindividual, withinperson approach to quantify the occurrence of marital transitions and their impact using data from the Health and Retirement Study ( $n=22,013 ; 1992-2010)$. Measures of
\end{abstract}

transition occurrence, depressive symptoms, and educational attainment were available across up to 10 biennial assessments. Results: Individuals with less than a high school diploma displayed the highest likelihood of losing their significant other through divorce/separation or death. Marital loss was associated with increasing, and marital gain with decreasing, depressive symptoms. Compared to those with less than a high school diploma, individuals with a high school or general equivalency diploma exhibited larger increases in depressive symptoms associated with widowhood, even though their average levels of depressive symptoms were lower in the absence of this transition. Conclusions: Our findings revealed a predictable educational gradient for the occurrence of marital transitions and laterlife mental health. Yet higher, formalized education did not protect the participants from increased depression in the presence of a loss-related transition, which could suggest that the strains of spousal loss may to some degree function as a leveler of the preexisting social inequalities of stratified life courses. We conclude that the benefits conferred by education are not necessarily ubiquitous, and its impact on the adaptation to spousal loss may be more complex and nuanced depending on the range of prior experiences and available coping strategies.

(c) 2018 S. Karger AG, Basel

\section{KARGER}

(c) 2018 S. Karger AG, Basel

E-Mail karger@karger.com

www.karger.com/ger
Claudia Recksiedler

Department for Social Monitoring and Methodology, German Youth Institute Nockherstrasse 2

DE-81541 Munich (Germany)

E-Mail recksiedler@dji.de 


\section{Introduction}

Later decades of the life course have undergone rapid transformations due to demographic changes in ageing societies, which affect the organization of all life domains such as family, work, and leisure [1]. As the average life span lengthens, particularly years without severe health and disability issues, individuals' potential to engage in meaningful and longer relationships with family members and other social ties expands as well [2]. However, the dramatic increase in rates of divorce and separation among older adults since 1980 has demonstrated that extended life expectancies also bring novel challenges in renegotiating social roles in older age, which could be a source of stress and emotional turmoil [3]. The ability to weather later-life marital transitions - whether more age normative, such as becoming widowed, or more novel, like divorcing - may especially depend on the long arm of education obtained earlier in the life course. For example, formal education is closely tied to the acquisition and mastery of resources, social ties, and coping strategies $[4,5]$.

Even though the effects of life events on depression are well documented [6], evidence for patterns of marital gain or loss and their association with depressive symptoms in old age, particularly within persons over time, is comparatively sparse. To address this research gap, this study used a within-person, intraindividual approach to studying marital transitions. The within-person approach, a hallmark of everyday stress research [7], leverages longitudinal data to track the occurrence of experiences (e.g., marital transitions) alongside variation in an individual's depressive symptoms to systematically test the association between when such experiences occur and the level of depressive symptoms. Marital transitions represent critical life events that may require coping and adaptation, temporarily or over an extended time span [8], and they are thus appropriate for a within-person approach. Lastly, because education is a key organizer of the life course and a protective factor against marital dissolution and depression $[9,10]$, we tested whether early-life educational attainment buffers the effect of marital transitions on depressive symptoms.

\section{Theoretical Considerations regarding Marital \\ Transitions in Old Age}

According to the life course perspective [11], human lives are organized by the timing, order, and interplay of social roles from birth to death. Transitions, such as getting married or losing a spouse, refer to discrete status changes in roles that structure life courses and form interconnected trajectories of life domains such as the family. Although individuals navigate and shape experiences related to role transitions agentically, their behaviors are embedded in and restricted by the sociohistorical context, as well as the choices of their closest social ties (e.g., close-knit kin or romantic partners). Particularly in middle and old age, individuals are part of an web of family ties consisting of spouses, children, grandchildren, siblings, and other confidants, which fulfill different emotional, social, and material needs. Any role transitions that alter highly complex family configurations in old age may therefore have far-reaching ripple effects on the mental health of the individual undergoing changes as well as their closest social ties $[6,12]$.

Different theoretical models have been formulated to conceptualize the effect of marital gain or loss as either a source of strength or strain for individuals' mental health. The marital resource model suggests that union formation is positively associated with mental health because couples share and pool each other's resources such as income and social ties [13]. In addition to emotional gains associated with marriage [14], older adults could benefit greatly from marriage because a sizeable part of older adults report shrinking social networks and - although to a lesser degree among younger cohorts - financial difficulties following spousal loss $[15,16]$. It is important to note, however, that partnering processes in old age are highly selective and complex $[10,17]$. Adjusting to life with a new partner or spouse may further induce stress associated with changes in daily routines and activities of the new couple, above and beyond the merging of complex family structures or the strain of caretaking needs for ageing spouses.

With regard to marital dissolution through divorce/ separation or widowhood, the crisis model is one approach to link spousal loss - reflecting a temporary life crisis - with detrimental mental health effects that fade over time [18]. Social selection along key sociodemographic characteristics, such as educational attainment, has further shown to play a role in the occurrence of marital loss [19] and its association with depression [20]. One could therefore speculate that mental health gaps between those who experience marital disruption and those who do not increase over time as a function of cumulative inequalities in the distribution of and access to resources fueled by initial group differences and stress proliferation (e.g., highly between individuals with low education) [21]. 
Alternatively, the transition as an equalizer approach predicts that educational gaps shrink as a consequence of transition occurrence because individuals with lower education are less vulnerable to event-related declines in these resources, given that they have often experienced economic deprivation and other stressors already before the critical life event [22]. As a result, there is little room for further loss for the less privileged and more to lose for their more privileged counterparts $[23,24]$.

\section{Marital Transitions and Mental Health in Old Age}

Marital transitions are more prevalent in earlier decades of adulthood; yet comprehensive demographic changes have transformed the composition of families in old age as well [1]. Average increases in individuals' life expectancy, particularly the prolonged time of being healthy and mobile [2], have coincided with declining rates of widowhood and rising divorce rates since the 1980s. The rate of divorced or separated older adults, for example, grew substantially from $5.3 \%$ in 1980 to $11.9 \%$ in 2009 [19], which has far-reaching implications for partner markets in this age group as well [17]. In contrast, the rate of widowed older adults aged 65 years and over shrank from $51.0 \%$ in 1980 to $39.9 \%$ in 2010 for women and from $13.6 \%$ in 1980 to $12.7 \%$ in 2010 for men [25]. Nevertheless, the distribution of marital transitions follows a social gradient in a way that low-socioeconomicstatus (SES) groups report marital loss more and marital gain less frequently $[10,19,26]$. In addition to well-documented education-related patterns of disease and mortality that may fuel group differences in rates of widowhood $[5,9]$, divorce rates are highest among unemployed individuals, non-Whites, those with lower levels of education, and remarried couples [19]. The rate of married older adults aged 65 years and over declined slightly from $75.5 \%$ in 1980 to $71.7 \%$ in 2010 for men and increased from $38.0 \%$ in 1980 to $42.4 \%$ in 2010 for women [25]. Whereas better-educated and healthier individuals report a stronger desire to remarry after having experienced spousal loss [27], only older adults' wealth could be linked to higher chances of cohabiting or marrying [10]. Sex ratio imbalances in old age further favor men's chances of marrying over women's [25].

Close relationships with others are an important source of meaning and happiness across the life course, and in old age particularly; yet they can also be a source of stress at the same time [3]. Changes in marital status, whether negative or positive, may be particularly critical for older adults' mental health as older adults tend to assume fewer social roles (e.g., after retiring from work) and a sizable proportion of older adults has strongly familycentered social networks [15]. Experiencing marital loss through the death of one's spouse has therefore been linked to heightened risks of depressive symptoms, loneliness, substance abuse, nursing home admission, or even one's own death, particularly for men and individuals with little social support $[13,28-31]$. Nonetheless, the majority of bereaved individuals - particularly women seem to successfully adapt to spousal loss over time, and only a minority continue to suffer from enduring psychological impairments [32]. This could be the case because widowhood is a more age-normative life event for older women due to gender differences in average life expectancies, combined with the fact that women tend to cultivate stronger social ties that may subsequently help them weather this transition. Going through a divorce or separation has been associated with a reduction in one's health similar to that with the loss of a partner through death and can bring additional social isolation or economic hardship [20,30]. This may further exacerbate preexisting inequalities in mental health for low-SES groups. Leopold and Leopold [23], however, demonstrated that divorce is not as consequential for women with lower education as for those with higher levels of educational attainment in younger age groups, because the latter group may have more to lose.

Blending families in old age becomes increasingly complex with children and grandchildren in the equation, and it may involve at least temporary strains [12]. Nevertheless, remarriage seems to foster higher levels of well-being through love and companionship and may offset potential health impairments from previous marital loss $[14,30]$. It is important to note, however, that even loss-related marital transitions may have positive spillover effects on individuals' mental health under certain circumstances. For example, leaving a troubled marriage preceded by chronic conflicts reduces stress and depressive symptoms $[13,20]$, and being relieved from longterm caretaking duties for a disabled or sick spouse has also been linked to a quicker adaptation to the new role as widow/er [29]. Taken together, marital transitions represent diverse experiences that contribute to mental health in later life [33], and their effects could potentially be augmented by existing SES-related disparities in mental health [34].

\section{The Present Study}

Patterns of marital transition have become more complex in older populations. Despite considerable research linking life events to depression, less is known about mar- 
ital transition-depression linkages during old age and within persons over time and their variation along key sociodemographic characteristics such as educational attainment. More specifically, using large-scale longitudinal data and analytical methods suitable to examine within-person change and variation, we aimed to prospectively track marital transitions and their impact on mental health within persons over time. This method further circumvents the potential measurement biases related to commonly used approaches that quantify the impact of critical life events with retrospective checklists or between-group comparisons by marital status (e.g., continuously married vs. widowed vs. divorced), permitting examination of the extent to which fluctuations in individuals' depressive symptoms are yoked to the occurrence of marital transitions. In doing so, the present study is addressing this research gap by examining three research aims.

First, we aimed to identify and track the occurrence of the marital transitions of marriage, divorce/separation, and widowhood experienced among older adults. We quantified the occurrence of marital transitions using up to 18-year biennial longitudinal data to document transition occurrence across time. Based on prior research, we expected individuals with lower levels of educational attainment to be more likely to experience spousal loss through divorce/separation and death, yet less likely to marry (hypothesis 1).

Second, we aimed to link the occurrence of marital transitions with depressive symptoms within persons over time, since these transitions represent life events that may increase the need for coping and adaption. We predicted that the occurrence of divorce and widowhood would be associated with an increase in depressive symptoms within persons over time (hypothesis $2 \mathrm{a}$ ). For marriage, however, we expected a decrease in depressive symptoms despite a period of adjustment to this new role (hypothesis 2b).

Third, we aimed to test the influence of educational attainment on the link between marital transition and depression. Against the theoretical and empirical backdrop, we formulated two competing working hypotheses. According to the "cumulative disadvantage" perspective and models of stress adaptation, we expected higher educational attainment to shield individuals from the impact of marital transition on depressive symptoms (hypothesis 3a). According to the "transition as an equalizer" perspective, however, we could also expect that higher educational attainment would not buffer but might exacerbate the impact of marital transition on depressive symptoms (hypothesis 3b). More highly educated individuals may display larger increases in depressive symptoms following marital transitions than their peers with lower education for two potential reasons: on the one hand, those with a more privileged social position conferred by education may have more to lose in light of spousal loss; on the other hand, they may also be less resistant because they have faced fewer stressors prior to the transition.

\section{Methods}

Data

We drew data from two longitudinal studies of nationally representative probability samples: the initial cohorts of the Health and Retirement Study (HRS) and the Study of Assets and Health Dynamics among the Older Old (AHEAD). Both studies sampled households of noninstitutionalized individuals over the age of 50 years in the contiguous USA. The initial full HRS cohort was assessed in 1992 and was composed of 12,654 adults between the ages of 50 and 60 years (born between 1931 and 1941) from 7,704 households. The initial full AHEAD cohort was assessed in 1993 and comprised 8,222 adults aged 70 years and older (born on or before 1923) from 6,046 households. Data from the HRS and AHEAD were collected every 2 years using mixed-mode assessments (i.e., face-to-face and telephone interviewing), and the studies were merged into a single interview schedule in 1998.

For our study, we further included the HRS cohort Children of the Depression (CODA; born between 1924 and 1930) and the refresher cohort War Babies (WB; born between 1942 and 1947) in our sample, which had both been added to the HRS in 1998. Our final analytic sample consisted of all available information across the study waves 1992-2010 (up to 10 waves of biennial assessments) for the 4 study cohorts, excluding only study waves where responses were given by proxy respondents $(n=22,013)$.

\section{Measures}

Marital Transitions. Changes in marital status (marriage, divorce/separation, and widowhood) were measured using data from the biennial core interviews of the HRS. The participants were asked to state their current marital status during each assessment (e.g., married, widowed, separated, or divorced). In some HRS waves, divorce and separation were distinct answer categories, whereas they were combined in others. For the purpose of this study, we created a single variable for divorce/separation because it was impossible to disentangle divorced and separated individuals consistently across the study waves. Based on this information, we generated a dichotomous variable indicating whether each of the events was experienced at a particular wave by comparing the current-wave marital status to the previous-wave marital status to quantify marital transitions. Thus, if the marital status across adjacent waves was incongruent, the current-wave status served as the index of transition occurrence (i.e., became married, widowed, or divorced/separated). Note that the marital transitions at each wave were mutually exclusive.

Depressive Symptoms. Depressive symptoms were measured using an 8-item variant of the Center for Epidemiological Studies Depression Scale (CES-D) [35], developed for the HRS. The CES- 
Table 1. Descriptive statistics of the study cohorts in the final analytic sample

\begin{tabular}{|c|c|c|c|c|c|}
\hline Indicator & HRS & AHEAD & CODA & WB & Total \\
\hline \multicolumn{6}{|l|}{ At baseline } \\
\hline Subjects, $N(\%)$ & $10,587(48.1)$ & $7,266(33.0)$ & $2,175(9.9)$ & $1,985(9.0)$ & $22,013(100.0)$ \\
\hline Mean age (SD), years & $56.30(4.32)$ & $76.33(6.32)$ & $70.54(2.08)$ & $53.06(2.61)$ & $64.03(10.81)$ \\
\hline Male, $n(\%)$ & $5,179(48.9)$ & $2,563(35.3)$ & $859(39.5)$ & $1,009(50.8)$ & $9,610(43.7)$ \\
\hline \multicolumn{6}{|l|}{ Education, $n(\%)$} \\
\hline Less than high school & $2,885(27.2)$ & $2,998(41.3)$ & $611(28.1)$ & $266(13.4)$ & $6,760(30.7)$ \\
\hline At least some college & $2,131(20.2)$ & $953(13.1)$ & $436(20.0)$ & $651(32.8)$ & $4,171(19.0)$ \\
\hline \multicolumn{6}{|l|}{ Race, $n(\%)$} \\
\hline Caucasian & $8,494(80.2)$ & $6,206(85.6)$ & $1,909(87.8)$ & $1,605(80.9)$ & $18,214(82.8)$ \\
\hline African American & $1,804(17.0)$ & $957(13.2)$ & $205(9.4)$ & $320(16.1)$ & $3,286(14.9)$ \\
\hline Other & $289(2.8)$ & $87(1.2)$ & $60(2.8)$ & $60(3.0)$ & $496(2.3)$ \\
\hline Mean depression score (SD) & $2.18(1.98)$ & $1.66(2.00)$ & $1.36(1.77)$ & $1.43(1.87)$ & $1.86(1.98)$ \\
\hline \multicolumn{6}{|l|}{ Pooled across waves } \\
\hline Widowed & $1,650(16.4)$ & $1,477(24.3)$ & $373(18.8)$ & $125(6.6)$ & $3,791(18.1)$ \\
\hline Married & $451(4.5)$ & $88(1.4)$ & $41(2.1)$ & $97(5.1)$ & $677(3.4)$ \\
\hline Divorced/separated & $744(7.4)$ & $188(3.1)$ & $96(4.8)$ & $137(7.2)$ & $1,165(5.8)$ \\
\hline Mean time in study ${ }^{\mathrm{a}}(\mathrm{SD})$ & $4.98(2.87)$ & $3.61(2.31)$ & $3.54(2.00)$ & $3.82(2.01)$ & $4.43(2.69)$ \\
\hline \multicolumn{6}{|c|}{ 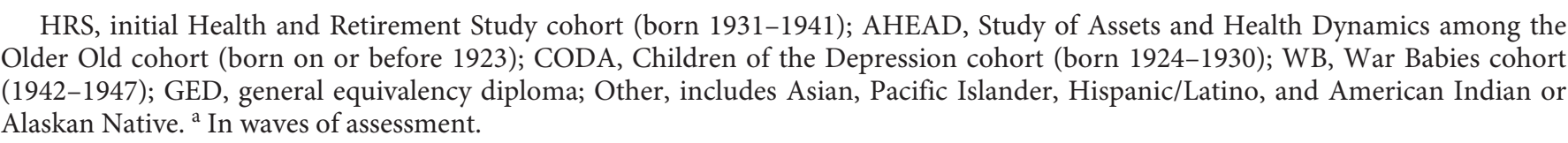 } \\
\hline
\end{tabular}

D was constructed to measure a continuum of psychological distress by asking respondents to state whether they felt symptoms of depression much of the time during the previous week ( $1=$ yes; $0=$ no). A count variable ranging from 0 to 8 was formed based on individuals' rating of the 8 items, such as, "Much of the time during the past week, I felt that everything I did was an effort." This modified, shortened scale has shown to have a good internal consistency ( $\alpha=0.81-0.83$ ), a factorial structure similar to the full scale, and a good construct validity [36].

Educational Attainment. Early-life educational attainment at baseline was measured by asking respondents to report their highest grade of school or year of college completed (in full years), as well as to state their highest degree earned $(0=$ no formal degree; $1=$ high school equivalency [general equivalency diploma (GED)]; 2 = high school diploma; $3=2$-year college; $4=4$-year college; $5=$ master's degree; 6 = professional degree [e.g., doctor's degree, etc.]; $7=$ degree unknown/some college). Given the smaller proportion of respondents with a higher degree and relevant educational levels established in previous research, we grouped the respondents' highest degree earned into a 3 -level variable $(0=$ less than high school diploma; 1 = high school diploma or GED; 2 = some college or more), which was subsequently used in all models as a predictor and effect modifier (see below).

Control Variables. Information about the participants' chronological age at baseline (in full years), study cohort ( 0 = initial HRS; 1 = AHEAD; $2=\mathrm{CODA} ; 3=\mathrm{WB})$, gender $(0=$ female; $1=$ male $)$, race $(0=$ Caucasian; $1=$ African American; $2=$ other, such as
Asian, Pacific Islander, Hispanic/Latino, and American Indian or Alaskan Native), and time in study (count of present study waves) was available at baseline. These indicators served as control variables in all analyses to adjust for minor differences in the sociodemographic composition of the sample. We further used the respondents' self-rated health (single-item indicator: from $1=$ poor health to $5=$ excellent health) as a time-varying control variable to adjust for potential selection effects in the occurrence and impact of marital transitions.

\section{Analytic Strategy}

All statistical analyses were conducted using Stata (version 13.1). The initial analyses included descriptive statistics to determine the prevalence and distribution of marital transitions and depression indices at the individual, subgroup, and cohort levels (Table 1). To examine differences and changes in the likelihood of experiencing marital transitions over the study period (aim 1; hypothesis 1), we used logistic multilevel models that were adjusted for time in study, age at baseline, cohort, gender, race, educational attainment, and self-rated health (Stata command xtmelogit). As self-rated health was a time-varying variable (i.e., assessed at each wave), we employed person-mean centering [37], and included both the person-mean and person-specific deviation score to covary for between- and within-person variation in self-rated health. To test the time-varying relationship between marital transitions and depressive symptoms (aim 2; hypotheses $2 \mathrm{a}$ and $2 \mathrm{~b}$ ), we used mixed-effects multilevel models (Stata command mixed). First, we 
Table 2. Distribution of the available data across the waves and study cohorts in the sample in $n$ (\%)

\begin{tabular}{llllll}
\hline Wave & HRS & AHEAD & CODA & WB & Total \\
\hline 1 & $10,587(100.0)$ & $7,266(100.0)$ & $2,175(100.0)$ & $1,985(100.0)$ & $22,013(100.0)$ \\
2 & $9,375(88.5)$ & $7,266(80.6)$ & $1,900(87.4)$ & $1,773(89.3)$ & $18,908(0.86)$ \\
3 & $8,779(82.9)$ & $5,860(66.0)$ & $1,688(77.6)$ & $1,702(85.7)$ & $16,965(0.77)$ \\
4 & $8,191(77.4)$ & $4,796(54.2)$ & $1,543(70.9)$ & $1,643(82.8)$ & $15,314(0.70)$ \\
5 & $7,600(71.8)$ & $3,937(43.2)$ & $1,397(64.2)$ & $1,595(80.3)$ & $13,731(0.62)$ \\
6 & $7,224(68.2)$ & $2,568(35.3)$ & $1,251(57.5)$ & $1,543(77.7)$ & $12,586(0.57)$ \\
7 & $6,960(65.7)$ & $2,061(28.4)$ & $1,027(47.2)$ & $1,491(75.1)$ & $11,539(0.52)$ \\
8 & $6,687(63.2)$ & $1,586(21.8)$ & & & $8,273(0.38)$ \\
9 & $6,307(59.6)$ & $1,011(13.9)$ & & & $7,318(0.33)$ \\
10 & $5,708(53.9)$ & & & & $5,708(0.26)$ \\
\hline
\end{tabular}

Blank cells represent missingness by design. HRS, initial Health and Retirement Study cohort (born 19311941); AHEAD, Study of Assets and Health Dynamics among the Older Old cohort (born on or before 1923); CODA, Children of the Depression cohort (born 1924-1930); WB, War Babies cohort (1942-1947).

Table 3. Logistic multilevel regression results predicting marital transition occurrence

\begin{tabular}{|c|c|c|c|c|c|c|}
\hline \multirow[b]{2}{*}{ Predictors } & \multicolumn{2}{|c|}{ Widowhood } & \multicolumn{2}{|c|}{ Marriage } & \multicolumn{2}{|c|}{ Divorce/separation } \\
\hline & OR & $\mathrm{CI}$ & OR & $\mathrm{CI}$ & OR & $\mathrm{CI}$ \\
\hline Age at baseline & 1.00 & $0.99,1.01$ & $0.93^{* * *}$ & $0.91,0.95$ & $0.97 * * *$ & $0.95,0.98$ \\
\hline Time in study & $1.09 * * *$ & $1.07,1.11$ & $0.95 * *$ & $0.91,0.98$ & $0.97^{*}$ & $0.94,0.99$ \\
\hline Male & $0.56^{* * *}$ & $0.52,0.61$ & $1.59^{* * *}$ & $1.33,1.87$ & 0.89 & $0.77,1.02$ \\
\hline High school/GED ${ }^{a}$ & $0.81^{* * *}$ & $0.75,0.88$ & 0.82 & $0.66,1.02$ & $0.63^{* * *}$ & $0.54,0.74$ \\
\hline College $^{\mathrm{a}}$ & $0.69^{* * *}$ & $0.61,0.77$ & 0.94 & $0.72,1.22$ & $0.61^{* * *}$ & $0.50,0.76$ \\
\hline African American ${ }^{\mathrm{b}}$ & $1.24^{* * *}$ & $1.13,1.36$ & $1.33^{*}$ & $1.06,1.69$ & $2.02^{* * *}$ & $1.71,2.38$ \\
\hline Other $^{\mathrm{b}}$ & 1.06 & $0.83,1.37$ & 1.14 & $0.65,2.02$ & $2.08^{* * *}$ & $1.43,3.02$ \\
\hline $\mathrm{AHEAD}^{\mathrm{c}}$ & $2.30^{* * *}$ & $1.97,2.68$ & $1.94^{* *}$ & $1.24,3.05$ & 0.97 & $0.68,1.37$ \\
\hline $\mathrm{CODA}^{\mathrm{c}}$ & $1.78^{* * *}$ & $1.52,2.08$ & $2.02^{* *}$ & $1.27,3.22$ & $1.61^{* *}$ & $1.17,2.21$ \\
\hline $\mathrm{WB}^{\mathrm{c}}$ & $0.60^{* * *}$ & $0.49,0.75$ & 1.07 & $0.80,1.43$ & 0.95 & $0.73,1.23$ \\
\hline Self-rated health (BP) & $0.94^{* *}$ & $0.90,0.98$ & $1.19^{* *}$ & $1.08,1.32$ & $0.83^{* * *}$ & $0.77,0.90$ \\
\hline Self-rated health (WP) & 1.03 & $0.97,1.08$ & 1.07 & $0.93,1.22$ & 0.96 & $0.87,1.05$ \\
\hline
\end{tabular}

OR, odds ratio; CI, 95\% confidence interval; GED, general equivalency diploma; Other, includes Asian, Pacific Islander, Hispanic/Latino, and American Indian or Alaskan Native; AHEAD, Study of Assets and Health Dynamics among the Older Old cohort (born on or before 1923); CODA, Children of the Depression cohort (born 1924-1930); WB, War Babies cohort (1942-1947); BP, between persons; WP, within person. ${ }^{a}$ Reference category: less than a high school diploma. ${ }^{\mathrm{b}}$ Reference category: Caucasian. ${ }^{\mathrm{c}}$ Reference category: initial Health and Retirement Study cohort. ${ }^{*} p<0.05,{ }^{* *} p<0.01,{ }^{* * *} p<0.001$.

estimated an "empty" model for the unadjusted, overall trajectory of depressive symptoms (model 1). Next, we examined the timevarying marital transition predictors, again adjusting for all covariates listed above (model 2). Subsequently, we extended the multilevel models testing aim 2 by including respective educational attainment $\times$ marital transition interaction terms (model 3; hypotheses $3 \mathrm{a}$ and $3 \mathrm{~b}$ ). All models were estimated using maximum likelihood estimation.

Additional analyses examining pairwise comparisons of marital transition effects between and within educational categories were conducted using the lincom Stata command. Finally, to translate the effect of marital transitions on depressive symptoms into standard deviation (SD) units, we divided the slope estimates of marital transition effects on depressive symptoms by the within-person SD for depressive symptoms. The resulting values recast the transition effects as a proportion of the within-person variation (SD) in depressive symptoms, which served as an effect size measure.

As with any longitudinal study, particularly of older adults, attrition exists and could potentially threaten and/or bias the results. 
Table 4. Multilevel regression results predicting depressive symptoms

\begin{tabular}{|c|c|c|c|c|c|c|}
\hline \multirow[b]{2}{*}{ Predictors } & \multicolumn{2}{|l|}{ Model 1} & \multicolumn{2}{|l|}{ Model 2} & \multicolumn{2}{|l|}{ Model 3} \\
\hline & $B$ & SE & $B$ & $\mathrm{SE}$ & $B$ & SE \\
\hline \multicolumn{7}{|l|}{ Fixed effects } \\
\hline Intercept & $1.64^{* * *}$ & 0.01 & $1.80^{* * *}$ & 0.03 & $1.81^{* * *}$ & 0.03 \\
\hline Age at baseline & & & 0.00 & 0.00 & 0.00 & 0.00 \\
\hline Time in study & & & $0.01^{* * *}$ & 0.00 & $0.01^{* * *}$ & 0.00 \\
\hline Male & & & $-0.35^{* * *}$ & 0.02 & $-0.35^{* * *}$ & 0.02 \\
\hline High school/GED ${ }^{a}$ & & & $-0.32^{* * *}$ & 0.02 & $-0.33^{* * *}$ & 0.02 \\
\hline College $^{\mathrm{a}}$ & & & $-0.42^{* * *}$ & 0.03 & $-0.42^{* * *}$ & 0.03 \\
\hline African American ${ }^{\mathrm{b}}$ & & & 0.02 & 0.03 & 0.02 & 0.03 \\
\hline Other ${ }^{\mathrm{b}}$ & & & 0.12 & 0.07 & 0.12 & 0.06 \\
\hline $\mathrm{AHEAD}^{\mathrm{c}}$ & & & 0.01 & 0.04 & 0.01 & 0.04 \\
\hline $\mathrm{CODA}^{\mathrm{c}}$ & & & -0.00 & 0.04 & -0.00 & 0.04 \\
\hline $\mathrm{WB}^{\mathrm{c}}$ & & & $0.09^{* *}$ & 0.03 & $0.10^{*}$ & 0.03 \\
\hline Self-rated health (BP) & & & $-0.86^{* * *}$ & 0.01 & $-0.86^{* * *}$ & 0.01 \\
\hline Self-rated health (WP) & & & $-0.30 * * *$ & 0.01 & $-0.31^{* * *}$ & 0.01 \\
\hline Widowed & & & $0.74^{* * *}$ & 0.02 & $0.62^{* * *}$ & 0.04 \\
\hline Married & & & $-0.18^{* *}$ & 0.06 & -0.11 & 0.11 \\
\hline Div./sep. & & & $0.27^{* * *}$ & 0.04 & $0.20^{* *}$ & 0.07 \\
\hline Widowed $\times$ high school/GED & & & & & $0.19^{* * *}$ & 0.05 \\
\hline Married $\times$ high school/GED & & & & & -0.17 & 0.14 \\
\hline Div./sep. $\times$ high school/GED & & & & & 0.15 & 0.10 \\
\hline Widowed $\times$ college & & & & & 0.13 & 0.07 \\
\hline Married $\times$ college & & & & & 0.04 & 0.17 \\
\hline Div./sep. $\times$ college & & & & & -0.06 & 0.13 \\
\hline \multicolumn{7}{|l|}{ Variance components } \\
\hline Random intercept & $1.98^{* * *}$ & 0.02 & $1.21^{* * *}$ & 0.02 & $1.21^{* * *}$ & 0.02 \\
\hline Residual variance & $1.93^{* * *}$ & 0.01 & $1.71^{* * *}$ & 0.01 & $1.71^{* * *}$ & 0.01 \\
\hline
\end{tabular}

Table 2 details the percentage of sample retention across the study cohorts and waves of assessment. To evaluate and assuage concerns about this, we conducted additional sensitivity analyses. First, we restricted our analysis to the $34.1 \%(n=7,551)$ of the participants who completed all respective study waves. The pattern and statistical significance of the results were unchanged (analyses not shown). Second, we employed pattern mixture models [38] to evaluate whether CES-D scores or the effects of the family transitions on CES-D scores varied as a function of the number of waves of assessment. While earlier attrition was associated with significantly higher CES-D scores $(p=0.001)$, neither family transition effects (all $p s>0.26$ ) nor education $\times$ family transition interaction effects therein varied as a function of the number of waves of assessment (all ps > 0.37). Taken together, the current results appear to be robust to missingness due to attrition.

Marital Transitions and Depression

\section{Results}

\section{Marital Transition Occurrence among Older Adults}

The summary statistics of the sample and key study variables are presented in Table 1 . The older cohorts, AHEAD and CODA, consisted of more than $60 \%$ female respondents, whereas the younger cohorts had lower percentages of female respondents (e.g., 50.8\% in the WB cohort). The participants in the younger cohorts also tended to be more educated than those in the older cohorts. The proportion of individuals experiencing marriage or divorce at any point across the study period was relatively small across the cohorts, whereas $24.3 \%$ of the 


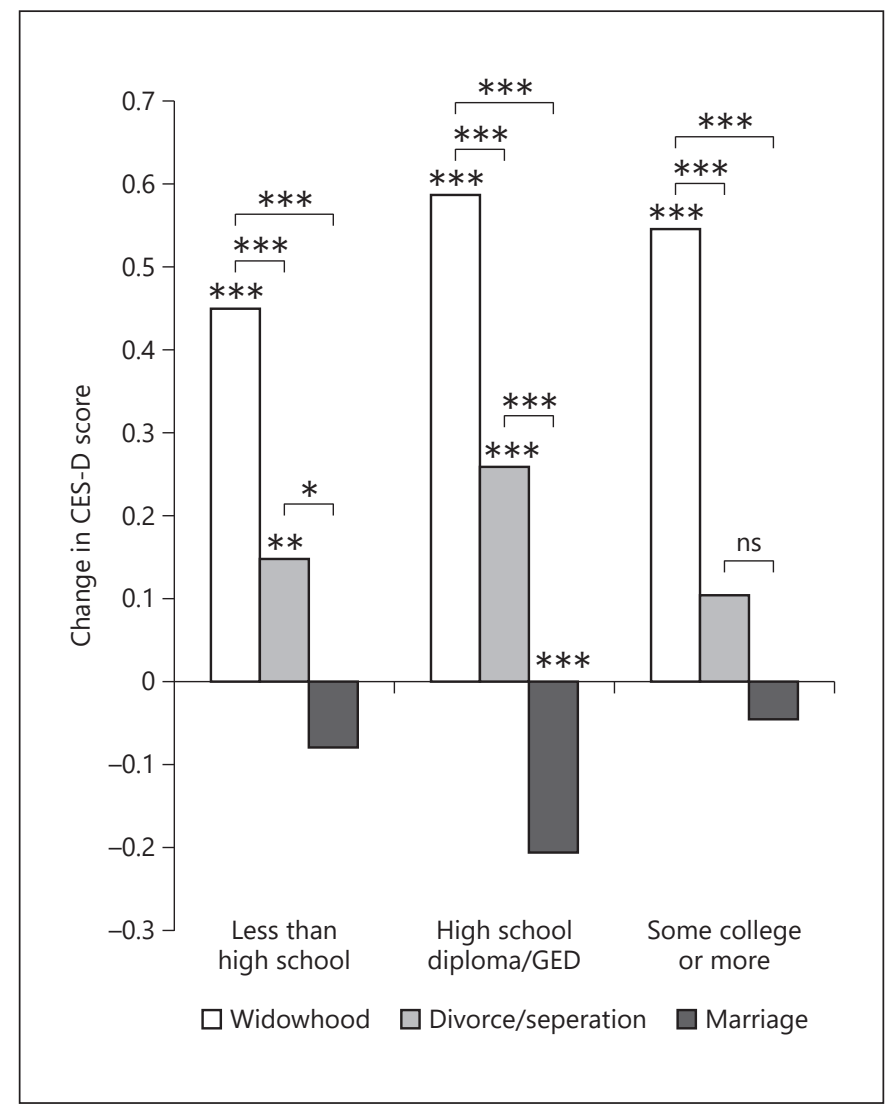

Fig. 1. Changes in depressive symptoms following marital transitions by educational attainment in standard deviation units. Brackets indicate a pairwise comparison transition effect within an educational category. ${ }^{*} p<0.05,{ }^{* *} p<0.01,{ }^{* * *} p<0.001$. CES-D, Center for Epidemiological Studies Depression Scale; GED, general equivalency diploma.

initial HRS cohort and $18.8 \%$ of the CODA cohort were widowed.

The results from the logistic multilevel models predicting the likelihood of each marital transition are summarized Table 3. Compared to the respondents with less than a high school diploma, the odds of experiencing a divorce/ separation or becoming widowed were significantly lower for those with a high school education $(\mathrm{OR}=0.63, p<$ 0.001 , and $\mathrm{OR}=0.81, p<0.001$, respectively) and those with some college or more (OR $=0.61, p<0.001$, and $\mathrm{OR}=0.69, p<0.001$, respectively). For instance, the odds of experiencing a divorce/separation at any given wave were $1 / 0.63=1.59$ times lower for those with a high school education than for those with less than a high school diploma. No significant differences in educational attainment emerged for marriage. Hypothesis 1, which stated that individuals with lower education would be more likely to experience marital loss and less likely to experience marital gain, was therefore only partially supported.

\section{Depressive Symptoms and Marital Transitions}

Table 4 displays the multilevel regression models with time-invariant and -varying predictors testing the association between depressive symptoms and marital transitions. Model 2 showed that when widowhood or divorce/ separation occurred, depressive symptoms increased ( $B$ $[\mathrm{SE}]=0.74[0.02], p<0.001$, and $B[\mathrm{SE}]=0.27[0.04], p<$ 0.001 , respectively). In terms of effect sizes, becoming widowed was associated with a $0.53-S D$ increase, whereas divorce/separation was associated with a $0.19-S D$ increase, in depressive symptoms. The opposite pattern was observed for marriage $(B[\mathrm{SE}]=-0.18[0.06], p<0.01$ ), which resulted in a 0.13 -SD decrease in depressive symptoms. Thus, both hypothesis $2 \mathrm{a}$ on the negative effect of marital loss and hypothesis $2 b$ on the positive effect of marital gain on depressive symptoms were supported.

\section{Educational Differences in Depressive Symptoms following Marital Transitions}

Model 3 shows the results for adding interactions between educational attainment and marital transitions (Table 4). We found a significant interaction term for the transition to widowhood only. Compared to those with less than a high school diploma, the individuals with a high school education exhibited larger increases in depressive symptoms associated with widowhood $(B[\mathrm{SE}]=$ 0.19 [0.05], $p<0.001)$. Figure 1 , showing the slope estimates for each transition by educational attainment, further illustrates that changes in depressive symptoms following widowhood and divorce/separation were most pronounced for individuals with a high school diploma $(B[\mathrm{SE}]=0.81[0.03], p<0.001$, and $B[\mathrm{SE}]=0.36[0.06]$, $p<0.001$, respectively). More specifically, becoming widowed was associated with a 0.59 -SD increase and divorce/ separation with a $0.26-S D$ increase in depressive symptoms among those with a high school degree. Marriage, however, was significantly associated with a 0.21 -SD decrease in depressive symptoms only for this group as well $(B$ SE] $=-0.29[0.08], p<0.001)$. Our results therefore seem to at least partially support hypothesis $3 \mathrm{~b}$ on shrinking educational gaps according to the "transition as an equalizer" perspective, rather than the "cumulative disadvantage" perspective (hypothesis 3a).

To further discern educational differences in the adaptation to later-life marital transitions from a different angle, we asked whether, for any given educational group, 
the impact of one transition mattered more than the impact of another. We contrasted the impact of a given transition on depressive symptoms across the educational strata by conducting pairwise comparisons of the groupspecific slope estimates of the marital transitions from model 3 (e.g., slope of becoming widowed vs. slope of divorcing/separating among respondents with a high school diploma/GED) in additional analyses (Fig. 1). Note that the brackets in Figure 1 signal whether the difference between the slope estimates of two given transitions within an educational group was significant or not. The results indicate that, across all educational groups, widowhood seemed to have the strongest impact compared to the other two transitions. Again, the relative impact of two out of three marital transitions on depressive symptoms was most pronounced for individuals with a high school education: becoming widowed versus marrying $(B[\mathrm{SE}]=$ 1.10 [0.09], $p<0.001)$ and divorcing/separating versus becoming married $(B[\mathrm{SE}]=0.64[0.10], p<0.001)$. The increase in depressive symptoms after becoming widowed versus divorcing/separating, however, was largest among individuals with some college or more $(B[\mathrm{SE}]=$ $0.61[0.12], p<0.001)$.

\section{Discussion}

This study contributes to the literature on the prevalence and impact of later-life marital transitions on older adults' mental health, as well as the impact of early-life education on these relationships $[8,13]$. We used a within-person approach to quantify marital transition occurrence using longitudinal tracking over nearly 2 decades rather than relying on checklist data [33]. This approach allows the evaluation of older adults' depressive symptoms across time and in the context of experienced transitions, which circumvents problems associated with retrospective and cross-sectional study designs. With regard to loss-related marital transitions, our results are in line with our expectations and previous studies that documented educational disparities in the exposure to divorce/separation and widowhood [26]. Despite increased rates of divorce and separation among older individuals over the last decades [19], it still represents a relatively rare event in our sample compared to the more age-normative transition into widowhood.

The lack of educational differences in the likelihood of marrying, which was contrary to our expectations that individuals with lower education would be less likely to remarry, could be attributed to a general trend toward

Marital Transitions and Depression forming less formal unions as an alternative to marriage across the life course and in old age (e.g., dating, living apart together, or cohabitation) [17]. Even though bettereducated older adults have been shown to express a stronger desire to remarry [27], practical challenges in the merging of households, conflict with adult children, or the potential loss of social security benefits - at least until the age of 60 years, when the remarriage penalty is lifted - may outweigh the benefits of marriage for all educational groups.

Undergoing life events, such as marital transitions, impacts individuals' mental health - potentially both as a sign of a temporary crisis and as chronic distress $[6,18]$. We found an increase in depressive symptoms associated with the experience of widowhood or divorce/separation and decreasing depressive symptoms for marriage, which was in line with our expectations. The impact of marital transition on depression is not uniform across individuals, but rather varies across several dimensions such as the circumstances of the transition [32], gender [13], and protective factors fostering successful coping, such as educational attainment [5]. Our results indicated that better-educated individuals - particularly those with a high school education - were more severely affected by spousal loss through widowhood than those with less than a high school diploma. Yet, in the absence of these marital transitions, the better-educated participants reported less depression than the least-educated participants, which is in line with the well-documented link between educational attainment and related constructs such as social class and health [9].

These findings may be striking at first, particularly from a "cumulative disadvantage" perspective that would suggest that experiencing transitions can exacerbate preexisting inequalities [21]. However, the "transition as an equalizer" perspective and models of resilience to adversity could explain the findings [22-24]. Although higher levels of education tend to promote successful development and adaption across the life course in the absence of marital loss, the better-educated may have simply more to lose in terms of declines in resources when facing marital loss and may have had fewer experiences with prior stressors than their less-privileged peers. Similarly, Liker and Elder [39] reported that the marital quality of middleclass couples was more adversely affected by economic hardship during the Great Depression era than that of working class couples who had to cope with financial insecurities already before the recession. Thus, the effect of loss-related marital transitions may also be blunted because of lower-education individuals' cumulative or 
chronic exposure to adverse contexts and events across the life course, which may enable them to show a higher tolerance to adverse life events [8].

These transitions, particularly widowhood, are undoubtedly stressful for each individual, regardless of one's educational attainment. For example, when comparing the relative impact of marital transitions by educational attainment, we see a "dose-response" relationship where widowhood fosters the largest increase in depressive symptoms, followed by divorce/separation and marriage, across all levels of education. But given lower-education individuals' higher baseline depression scores, more highly educated individuals may experience larger declines in mental health, because they may have a lower threshold for what constitutes a disruptive transition. Alternatively, the nominally similar marital transitions experienced by individuals across educational groups may be qualitatively different [33]. In contrast, the participants with lower education may have adopted a set of established coping strategies for critical transitions, such as acceptance, reappraisal, or benefit finding, due to prior exposure to stressors that could help dampen and make them weather the impact of marital loss on depressive symptoms [24, 40]. Lastly, because spousal loss is also more prevalent, and potentially more normative, for groups with lower education $[19,26]$, these individuals may also have larger support networks of peers undergoing similar experiences. This could, in turn, prevent further increases in depressive symptoms for this group as well.

\section{Limitations and Future Directions}

Our study has several limitations. First, the measures of marital transition status are derived from the participants' biennial assessments of marital status only. It could be the case that we are underestimating the occurrence of marital transitions because multiple transitions could have occurred during the 2-year time span between assessments and only the most recent marital status was recorded. Furthermore, we did not consider "softer" family-related transitions such as cohabitation, dating, or living apart together as alternatives to marriage, which are on the rise for older adults as well [10]. Given the increasing but still low prevalence of marital transitions among older adults in other studies [1], we are confident that our estimates are reasonable and argue that continued research on the prevalence and distribution of marital transitions in older age is warranted.

Second, and relatedly, our measure of marital transition occurrence did not differentiate between first marriages and remarriages. It is possible that the respective associations could differ for respondents entering a first versus a higher-order marriage in old age, because those transitions may represent quantitatively and qualitatively distinct experiences. Nevertheless, it is highly likely that most of the marriages recorded in our study classified as remarriages given the age group.

Third, domain-specific trajectories are intertwined [11]. Yet, we did not examine the dynamic interplay of the occurrence of transitions in other domains and its impact on depressive symptoms. It could be the case that the accumulation of transitions across domains overburdens individuals' capacity to cope with these changes at once, which in turn exacerbates the rise in depressive symptoms. The inclusion of transitions across multiple domains, however, was beyond the scope of this study and should be addressed in future research.

Fourth, we did not evaluate a potential anticipatory period leading up to spousal loss or whether widowhood or divorce/separation occurred rather suddenly, which may influence the adaption process. For example, the loss of a spouse is related to less steep declines in life satisfaction if there has been a long anticipatory period (e.g., in the case of long-term illness) [29]. Future research could benefit from leveraging such complementary approaches to provide more nuanced considerations of life event/ transition-health links during later life.

Lastly, our pattern of results may not apply to other dimensions of SES, such as social class or poverty, because we focused solely on measures of educational attainment. Nevertheless, education is an important organizer of the life course and has shown to be a consistent predictor of health disparities [4], and our results are consistent with perspectives on resilience and persistence in the face of adversity among low-SES populations [24].

\section{Conclusions}

We showed a predictable education gradient in the experience of marital transitions among older adults and their average levels of depressive symptoms. Yet, the education differences in the impact of such transitions do not follow a similar gradient. This may suggest that the benefits conferred by higher education and the vulnerabilities conferred by lower education are not necessarily ubiquitous. They may be more complex and nuanced depending on the type of transition, its timing in the life course, prior exposure to stress, and available coping strategies. Finally, the lack of a buffering effect of early-life educational attainment in the association between spousal loss and de- 
pression suggests that the universally demanding experience of spousal loss may, to some degree, serve as a leveler of the preexisting social inequalities of stratified life courses. Nevertheless, this mechanism may be specific to the study cohorts, for which remaining in post-secondary education for extended time periods was rather rare, and will need to be examined for other birth cohorts from the USA and other nations. Because marital biographies across the life course have become more complex - both for people now in later life and for future elders who are now in midlife and early adulthood - more studies will need to address the dynamic interplay between education (and other forms of social stratification) and the adaptation to marital transitions.

\section{Acknowledgements}

This work was supported by a grant from the National Institute on Aging (NIA) (R03-AG042919-01) and a Pilot Grant from the Michigan Center on the Demography of Aging (P30-AG012846).

\section{Statement of Ethics}

The authors have no ethical conflicts to disclose.

\section{Disclosure Statement}

The authors report no conflicts of interest.

\section{References}

1 Manning WD, Brown SL. The demography of unions among older Americans, 1980-present: a family change approach. In: Settersten RA Jr, Angel JL, editors. Handbook of Sociology of Aging. New York: Springer; 2011. p. 193-210.

2 Martin LG. Demography and aging. In: Binstock RH, George LK, editors. Handbook of Aging and the Social Science. San Diego: Elsevier Academic Press; 2011. p. 33-56.

3 Walen HR, Lachman ME. Social support and strain from partner, family, and friends: costs and benefits for men and women in adulthood. J Soc Pers Relat. 2000;17(1):5-30.

4 Miroswsky J, Ross CE. Education, Social Status and Health. New Brunswick: Aldine; 2003.

5 Whitfield KE, Thorpe R, Szanton S. Health disparities, social class, and aging. In: Schaie KW, Willis SL, editors. The Handbook of the Psychology of Aging. Burlington: Elsevier; 2011. p. 207-18.

6 Kessler RC. The effects of stressful life events on depression. Annu Rev Psychol. 1997; 48(1):191-214

7 Almeida DM. Resilience and vulnerability to daily stressors assessed via diary. Curr Dir Psychol Res. 2005;14(2):64-8.

8 Pearlin LI. The life course and the stress process: some conceptual comparisons. J Gerontol B Psychol Sci Soc Sci. 2010 Mar;65B(2): 207-15.

9 Marmot M. Social determinants of health inequalities. Lancet. 2005 Mar;365(9464):1099_ 104.

10 Vespa J. Union formation in later life: economic determinants of cohabitation and remarriage among older adults. Demography. 2012 Aug;49(3):1103-25.
11 Elder GH Jr, Shanahan MJ. The life course and human development. In: Lerner RM, editor. Theoretical models of human development. Handbook of Child Psychology. Hoboken: John Wiley \& Sons; 2006. vol. 1, p. 665715 .

12 de Jong Gierveld J, Peeters A. The interweaving of repartnered older adults' lives with their children and siblings. Ageing Soc. 2003; 23(2):187-205.

13 Williams K, Umberson D. Marital status, marital transitions, and health: a gendered life course perspective. J Health Soc Behav. 2004 Mar;45(1):81-98.

14 Frech A, Williams K. Depression and the psychological benefits of entering marriage. J Health Soc Behav. 2007 Jun;48(2):149-63.

15 Fiori KL, Antonucci TC, Cortina KS. Social network typologies and mental health among older adults. J Gerontol B Psychol Sci Soc Sci. 2006 Jan;61(1):25-32.

16 Perrig-Chiello P, Spahni S, Höpflinger F, Carr D. Cohort and gender differences in psychosocial adjustment to later-life widowhood. J Gerontol B Psychol Sci Soc Sci. 2016 Jul;71(4): 765-74.

17 Sassler S. Partnering across the life course: Sex, relationships, and mate selection. J Marriage Fam. 2010 Jun;72(3):557-75.

18 Amato PR. The consequences of divorce for adults and children. J Marriage Fam. 2000; 62(4):1269-87.

19 Brown SL, Lin IF. The gray divorce revolution: rising divorce among middle-aged and older adults, 1990-2010. J Gerontol B Psychol Sci Soc Sci. 2012 Nov;67(6):731-41.

20 Johnson DR, Wu J. An empirical test of crisis, social selection, and role explanations of the relationship between marital disruption and psychological distress: A pooled time-series analysis of four-wave panel data. J Marriage Fam. 2002;64(1):211-24.
21 Dannefer D. Cumulative advantage/disadvantage and the life course: cross-fertilizing age and social science theory. J Gerontol B Psychol Sci Soc Sci. 2003 Nov;58(6):S327-37.

22 Biblarz TJ, Raftery AE. The effects of family disruption on social mobility. Am Sociol Rev. 1993;58(1):97-109.

23 Leopold L, Leopold T. Maternal education, divorce, and change in economic resources: evidence from Germany (April 2016) [Internet]. SOEPpaper No. 836. Available from SSRN: https://ssrn.com/abstract=2778090.

24 Chen E, Miller GE. "Shift-and-persist" strategies: why low socioeconomic status isn't always bad for health. Perspect Psychol Sci. 2012 Mar;7(2):135-58.

25 U.S. Census Bureau. $65+$ in the United States: 2010. Washington (DC): U.S. Government Printing Office; 2014.

26 Hatch SL, Dohrenwend BP. Distribution of traumatic and other stressful life events by race/ethnicity, gender, SES and age: a review of the research. Am J Community Psychol. 2007 Dec;40(3-4):313-32.

27 Carr D. The desire to date and remarry among older widows and widowers. J Marriage Fam. 2004;66(4):1051-68.

28 Alpass FM, Neville S. Loneliness, health and depression in older males. Aging Ment Health. 2003 May;7(3):212-6.

29 Infurna FJ, Wiest M, Gerstorf D, Ram N, Schupp J, Wagner GG, et al. Changes in life satisfaction when losing one's spouse: individual differences in anticipation, reaction, adaptation, and longevity in the German Socio-Economic Panel Study (SOEP). Ageing Soc. 2017;37(5):899-934. 
30 Hughes ME, Waite LJ. Marital biography and health at mid-life. J Health Soc Behav. 2009 Sep;50(3):344-58.

31 Zivin K, Christakis NA. The emotional toll of spousal morbidity and mortality. Am J Geriatr Psychiatry. 2007 Sep;15(9):772-9.

32 Spahni S, Morselli D, Perrig-Chiello P, Bennett KM. Patterns of psychological adaptation to spousal bereavement in old age. Gerontology. 2015;61(5):456-68.

33 Dohrenwend BP. Inventorying stressful life events as risk factors for psychopathology: toward resolution of the problem of intracategory variability. Psychol Bull. 2006 May; 132(3):477-95.
34 Thoits PA. Stress and health: major findings and policy implications. J Health Soc Behav. 2010;51 Suppl:S41-53.

35 Radloff LS. The CES-D scale: A self-report depression scale for research in the general population. Appl Psychol Meas. 1977;1(3):385401.

36 Steffick DE. Documentation of affective functioning measures in the Health and Retirement Study. HRS/AHEAD Documentation Report 2000 [Internet]. Available from: http://hrsonline.isr.umich.edu/sitedocs/ userg/dr-005.pdf.
37 Hoffman L, Stawski RS. Person as Contexts: evaluating between-person and within-person effects in longitudinal analysis. Res Hum Dev. 2009;6(2-3):97-120.

38 Hedeker D, Gibbons RD. Application of random-effects pattern-mixture models for missing data in longitudinal studies. Psychol Methods. 1997;2(1):64-78.

39 Liker JK, Elder GH Jr. Economic hardship and marital relations in the 1930s. Am Sociol Rev. 1983 Jun;48(3):343-59.

40 Helgeson VS, Reynolds KA, Tomich PL. A meta-analytic review of benefit finding and growth. J Consult Clin Psychol. 2006 Oct; 74(5):797-816 